Recepción: 22 / 08 / 2018

Aceptación: 27 / 09 / 2018

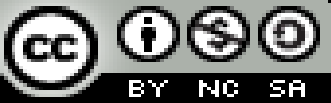

Ciencias económicas y empresariales

Publicación: 01 / 11 / 2018

Artículo de investigación

\title{
Gestión financiera en Tungurahua como paradigma en el liderazgo transformacional
}
Financial management in Tungurahua as a paradigm in transformational leadership
Gestão financeira na Tungurahua como paradigma da liderança transformacional

\author{
Jacqueline P. Hurtado-Yugcha I \\ jacquelinedhurtado@uta.edu.ec \\ Marcelo J. Mancheno-Saá II \\ mj.mancheno@uta.edu.ec \\ Jenny M. Gamboa-Salinas III \\ jgamboa12@yahoo.com \\ Alicia G. Ortiz-Morales IV \\ aliciagortiz@uta.edu.ec
}

\section{Correspondencia: jacquelinedhurtado@uta.edu.ec}

\footnotetext{
${ }^{\text {I } M a g i s t e r ~ e n ~ G e r e n c i a ~ F i n a n c i e r a ~ E m p r e s a r i a l, ~ I n g e n i e r a ~ d e ~ E m p r e s a s, ~ D o c e n t e ~ d e ~ l a ~ U n i v e r s i d a d ~ T e ́ c n i c a ~ d e ~ A m b a t o, ~ A m b a t o, ~}$ Ecuador.

II Docente de la Universidad Técnica de Ambato, Ambato, Ecuador.

III Magister en Gerencia Financiera Empresarial, Doctora en Ciencias Administrativas, Licenciada en Ciencias Administrativas, Docente de la Universidad Técnica de Ambato, Ambato, Ecuador.

${ }^{\text {IV }}$ Magister en Gerencia Financiera Empresarial, Magister en Pedagogía y Gestión Educativa, Doctora en Contabilidad y Auditoría, Licenciada en Contabilidad y Auditoria Contadora Publica, Docente de la Universidad Técnica de Ambato, Ambato, Ecuador.
} 


\section{Resumen}

El presente escrito tiene como propósito brindar orientaciones de la gestión financiera desde la perspectiva del liderazgo transformacional. Actualmente, en varias PYMES en Tungurahua se despliega un liderazgo rutinario que no se incluye a la realidad sociocultural y económico acoplado al entorno local. Actualmente las PYMES manejan un liderazgo tradicional y empírico lo cual se refleja en el manejo de la gestión financiera, mediante el crecimiento de activos e ingresos, fundamentados en esto, la metodología utilizada permitió analizar el comportamiento del sistema, en el cual se analizó cómo se comporta el objeto de estudio. Se pretende evaluar el funcionamiento del caso de estudio como es la gestión financiera en la realidad empresarial de la ciudad. En los resultados se evidencia que no todas las empresas presentan como base su sostenibilidad los activos y los ingresos, es importante determinar que no son manejadas bajo un proceso sinérgico que permita acceder a un desarrollo organizacional, en este sentido el liderazgo transformacional se presenta como un instrumento dinamizador de toda empresa que accede a nuevas oportunidades de negocio.

Con este artículo se espera apoyar la gestión financiera a través del necesario liderazgo organizacional adecuado a la realidad social, económica y cultural ecuatoriana.

Palabras clave: gestión financiera; liderazgo transformacional; empresa; PYMES; desarrollo organizacional.

\section{Abstract}

The purpose of this paper is to provide guidance on financial management from the perspective of transformational leadership. Currently, several SMEs in Tungurahua deploy a routine leadership that does not include the socio-cultural and economic reality coupled with the local environment. SMEs currently manage a traditional and empirical leadership which is reflected in the management of financial management, through the growth of assets and income, based on this, the methodology used allowed analyzing the behavior of the system, in which it was analyzed how it involves the object of study. It is intended to evaluate the operation of the case study as it is the financial management in the business reality of the city. The results show that not all companies present their assets and income as a basis for sustainability, it is important to determine that they are not managed under a synergistic process that allows access to an 
organizational development, in this sense the transformational leadership is presented as a dynamic instrument of any company that accesses new business opportunities.

With this article we hope to support financial management through the necessary organizational leadership appropriate to the Ecuadorian social, economic and cultural reality.

Key words: financial management; transformational leadership; company; SMEs; organizational development.

\section{Resumo}

O objetivo deste artigo é fornecer orientação sobre gestão financeira sob a perspectiva da liderança transformacional. Atualmente, várias PMEs em Tungurahua implantam uma liderança rotineira que não inclui a realidade sócio-cultural e econômica, juntamente com o ambiente local. Atualmente PME gerenciar liderança tradicional e empírica que se reflete em lidar com a gestão financeira, através do crescimento dos ativos e renda, com base nisso, a metodologia permitiu analisar o comportamento do sistema, que analisou como envolve o objeto de estudo. Pretende-se avaliar o funcionamento do estudo de caso, pois é a gestão financeira na realidade empresarial da cidade. Nos resultados, é evidente que nem todas as empresas têm a base de seus ativos de sustentabilidade e renda, é importante para determinar que eles não são geridos de acordo com um processo sinérgico que permite o acesso ao desenvolvimento organizacional nesse sentido liderança transformacional é apresentado como um instrumento dinâmico de qualquer empresa que acesse novas oportunidades de negócios.

Com este artigo esperamos apoiar a gestão financeira através da necessária liderança organizacional apropriada à realidade social, econômica e cultural equatoriana.

Palavras chave: gestão financeira; liderança transformacional; empresa; PME; desenvolvimento organizacional.

\section{Introducción}

En el proceso internacional de división del trabajo, la función del Ecuador y América Latina es la producción de materia prima de origen mineral o agropecuario para diferentes fines, basada principalmente en procesos extractivista; situación que históricamente ha mantenido a la 
economía ecuatoriana en desventaja, frente a los países con mayores niveles de industrialización, de los cuales importábamos la mayoría de productos con gran valor agregado y alta tecnología. Esta situación no ha cambiado durante décadas, nace entonces la necesidad de plantear una nueva estrategia de transformación económica productiva del país, basada en la diversificación productiva, agregación de valor de la producción existente, sustitución selectiva de importaciones y fomento de las exportaciones. (SENPLADES, 2012).

La diversificación productiva, se refiere a la determinación de industrias estratégicas, donde se intensifique los procesos de investigación y uso de tecnología, orientadas a maximizar la productividad en todos los eslabones de la cadena de valor, a la vez reducir la dependencia del país con enfoque de economía solidaria, dejando de lado los diferentes modelos históricos de acumulación.

La cosmopolita ciudad de Ambato, capital de la Provincia de Tungurahua emerge desde el centro del país como la urbe a nivel nacional con el mayor número de emprendedores de acuerdo al total de habitantes, esto se puede justificar con los datos del censo nacional económico y de población y vivienda (INEC 2010), en donde se observa que en la localidad el 25,33\% de su población son emprendedores es decir 3 de cada diez habitante tiene alguna actividad productiva propia, el $38,7 \%$ de la población económicamente activa (PEA) trabajan por cuenta propia, por tanto significa que no son buscadores, sino generadores de autoempleo, una menor proporción esto es el $32,4 \%$ son empleados privados y solo el 8.2\% trabajan para el estado (sector público). Esta información se contrasta con los datos de la encuesta nacional de empleo, desempleo e indicadores laborales del INEC a marzo 2015, en donde se aprecia que Ambato tiene una tasa de empleo adecuado del 53,97\%, de empleo inadecuado del 39,51\% (subempleo, otro empleo inadecuado y trabajadores no remunerados) y de desempleo del 6,43\%, es decir coincide con lo inicialmente dicho es decir 4 de cada 10 habitantes económicamente activos se encuentran en el estatus de subempleado porque por lo general sus emprendimientos son negocios personales o familiares que se desarrollan en un entorno con menor formalidad. (Proaño, 2015).

El liderazgo se establece como una herramienta dinámica en el progreso de las empresas, es importante que este liderazgo, no es solo el básico, el manejado bajo un proceso tradicionalista, sino que se enfoque a un cambio transformacional, el mismo que permita el acceso a nuevas 
oportunidades de negocio. De esta manera que la mejora de los resultados se basa en un proceso de cambio, en el que la transformación y las negociaciones se convierten en herramientas de trabajo, para lograr los objetivos de la organización y luego lograr un cambio significativo.

Por tanto, es más importante que este proceso pueda planificarse efectivamente, es decir, completar parámetros de administración organizacional. Desde este punto de vista, el liderazgo transformacional promoverá el desarrollo de una tarea eficiente en el manejo de su materialización en términos económico y financiero, entonces este proceso debe hacerse a través de una gestión óptima y transparente, para generar competitividad y confianza en la proyección de la organización hacia su sociedad.

\section{Fundamentos teóricos}

En la búsqueda del liderazgo asertivo se genera empoderamiento, de modo que se genera un trabajo integral, en el cual el enfoque sea orientado al empoderamiento organizacional y entonces proporcionar a las organizaciones una mejor eficiencia mediante una fuerza laboral creativa e innovadora, para esto el instrumento idóneo es el empoderamiento, el mismo que permite tomar decisiones sustentado en la capacidad del trabajo el cual se apoya en : a) poder otorgado al empleado, b) generación de motivación; c) acceso a capacitación y alto conocimiento de liderazgo. (Villar, 2012)

Es importante que los elementos de liderazgo indicados se agreguen al progreso personal y del experto líder, ya que será el desarrollo de sus habilidades y destrezas el instrumento principal para maximizar el desarrollo organizacional y luego estar en constante innovación en la alineación de recurso.

El nuevo liderazgo se enmarca entonces, en la innovación que permite el acceso a nuevas maneras de trabajo, bajo este enfoque existen dos formas particulares contemporáneas como la transaccional y transformacional:

- El liderazgo transaccional. Se basa en la cooperación entre los actores empresariales, en la cual las dos partes reciben premios y castigos generando así dependencia laboral, por ende, como el 
líder transaccional satisface las necesidades del trabajador, lo ayuda a lograr sus objetivos, generando así compromiso, honestidad y diligencia.

- En tanto que el liderazgo transformacional. Va más allá del simple intercambio, promueve dinamismo. (Bozzi, 2012).

La mejora de los resultados se basa en un proceso de cambio, en el que la transformación y las negociaciones se convierten en herramientas de trabajo, para lograr los objetivos de la organización y luego lograr un cambio significativo.

De esta manera el atributo del liderazgo se sustenta en un alto perfil comunicativo, el cual promueve integración en cada miembro de la organización y así accede al cumplimiento de las metas empresariales.

El liderazgo está constantemente ligado al poder, la autoridad y la política. Entonces en el entorno empresarial, el liderazgo se desarrolla acorde al nivel de autoridad el mismo que permite un perfil competitivo de los subordinados.

En este sentido la gestión financiera es un punto neurálgico del manejo organizacional al cual se debe establecer parámetros de direccionamiento sistemáticos que dinamicen toda la gestión empresarial.

La gestión financiera es la actividad que se lleva a cabo en una organización y responsable de la planificación, organización, gestión, control, monitorear y coordinar toda la gestión de los recursos financieros, para generar más beneficios y / o resultados. (Block, Stanley B, 2010).

El objetivo es, para asegurar que la organización se desarrolle de manera efectiva, tomar mejores decisiones financieras y generar oportunidades de inversión para la organización

La administración financiera es responsable de la asignación de fondos para activos corrientes y activos fijos; es capaz de obtener la mejor combinación y financiar alternativas y desarrollar una política de dividendos apropiada en el contexto de los objetivos de la empresa.

Por tanto, en la gestión financiera existen herramientas que ayudan a efectuar un proceso sistemático del manejo de los recursos económicos de la empresa como lo es la planificación financiera y el presupuesto, lo que ayudara a fomentar un mejor trabajo organizacional. La 
planificación financiera es una práctica financiera que reúne procesos y herramientas para establecer en una empresa los objetivos económicos; teniendo en cuenta los medios disponibles y los necesarios para lograrlo. (Moreno, 2014).

La función de los presupuestos en la administración de una empresa se comprende mejor cuando los presupuestos están relacionados con los mismos fundamentos de la administración, es decir, las funciones administrativas: planificación, organización, coordinación, gestión y control. (Burbano, 2015).

La planificación y el control como funciones administrativas de gestión se especifican en el proceso de preparación del presupuesto. Además, la organización, coordinación y dirección permiten la asignación de recursos y la implementación de planes para alcanzar los objetivos. El proceso consiste en analizar la gestión de los recursos de capital de trabajo, las inversiones fijas y diferidas, así como las decisiones operativas y no operativas tomadas durante un período y su financiación para verificar el cumplimiento de las metas y los objetivos. (Fierro, 2015).

Se trata de determinar mediante la sostenibilidad financiera de la gestión en las empresas, una sinergia con el liderazgo para generar un efectivo rendimiento, con un nivel aceptable de riesgo, a través de la evaluación de la inversión. y sus resultados, los mismos que promuevan dinamización de recursos al más alto nivel.

\section{Materiales y métodos}

En el desarrollo del trabajo se utilizó la metodología de investigación económico- administrativa para encontrar una solución científica a un problema de carácter económico administrativo. 


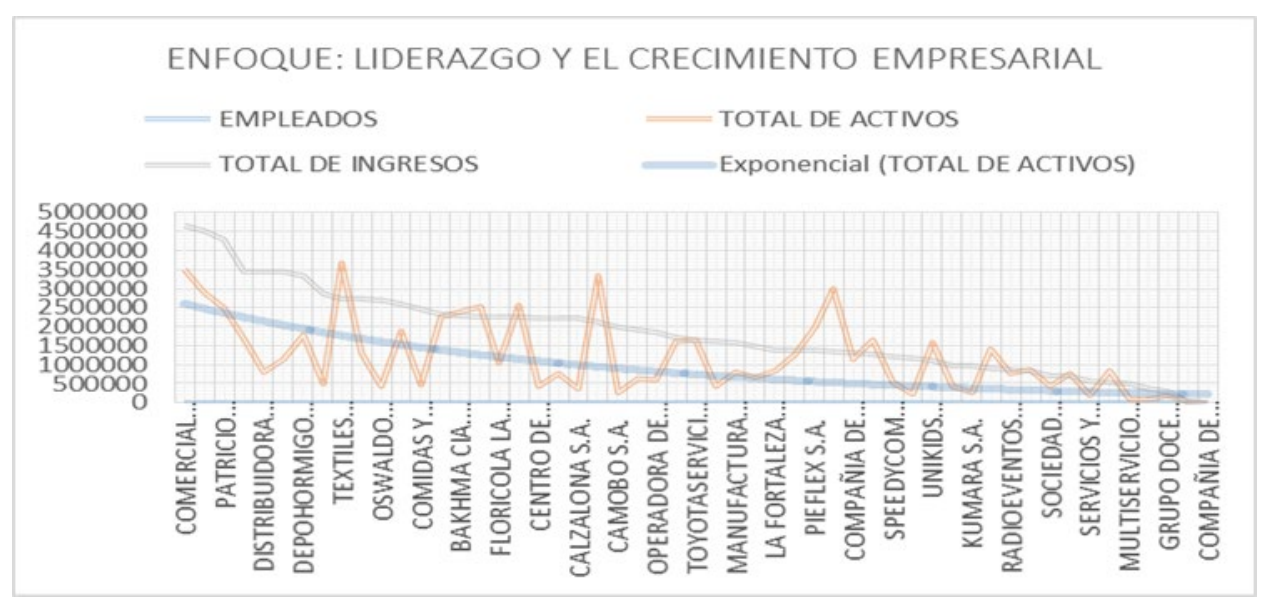

Fuente: Registro de la Superintendencia de Compañías- Tungurahua

También se utilizó la metodología de investigación con enfoque sistémico, que es un sistema es un conjunto de elementos íntimamente relacionados que pretenden satisfacer un objetivo común, en donde cada uno de sus elementos tiene una relación interactiva con los demás elementos, tanto internos como externos. (Bernal, 2013).

Al hacer referencia al manejo de la gestión financiera, se hace énfasis a un enfoque de carácter holístico con varios puntales integrales, en los cuales el liderazgo transformacional marca el paso para el desarrollo de los demás, el crecimiento empresarial tiene una estructura lógica indicando que el total de activos tiene una relación inversamente proporcional al total de ingresos, y que a su vez se relaciona con los colaboradores de la empresa. El grupo estudiado se trata de un grupo de empresarios de la ciudad de Ambato donde resaltan las PYMES representativas que cuentan con activos totales inferiores a cuatro millones de dólares y tienen más de 100 trabajadores.

Como se puede observar el sector de la PYMES en la localidad es representativo, de esta manera el crecimiento que han tenido ha sido basado en el liderazgo, el mismo que permite generar no solo un eficiente manejo de los recursos financieros, sino el acoplamiento de todos sus recursos para poder potencializar su perfil empresarial en el entorno. 


\section{Resultados}

En este contexto se determinó la necesidad de generar un cambio metodológico al modelo del liderazgo para que las PYMES mejoren su desarrollo en el mercado mediante un proceso sinérgico empresarial, por tanto, se establece que en el medio empresarial las empresas que han generado un cambio lo reflejan en su participación en el mercado: Con esta información se puede analizar que el crecimiento empresarial está basado en el liderazgo, el cual se refleja en la gestión financiera, en el manejo de sus recursos económicos, lo cual permite acceder a un mejor perfil empresarial.

\section{Conclusión}

Se determina entonces que la transición empresarial está enmarcada en el uso de instrumentos administrativos y financieros que permitan generar el acceso a nuevas oportunidades de negocio, de tal manera que el liderazgo gerencial enmarca en las bases de la integración de recursos, la cual bajo una sistematización de procesos se podrá acceder a nuevos mercados, donde las oportunidades de negocio promoverán una imagen de eficiencia empresarial en el entorno.

\section{Referencias Bibliográficas}

Bernal, Augusto. (2013). En Metodología de la Investigación. México: Pearson.

Block, Stanley B. (2010). En Fundamentos de la gerencia financiera. Bogotá: Mc Graw Hill.

Bozzi, S. M. (2012). En Liderazgo para el Cambio Social: Contribuciones para la teoría contemporánea sobre el liderazgo como construcción social. New York: New York University.

Burbano, Jorge E. (2015). Presupuestos. México: Trillas.

Fierro Martínez, Ángela María. (2015). En Administración Financiera. México: Mc Graw Hill. Moreno, Joaquín. (2014). En Planificación Financiera. México: Patria.

Proaño, D. (2015). Ambato el motor de los precios. Revista Ambato.

SENPLADES. (2012). En Transformación de la Matriz Productiva. Quito: Ediecuatorial.

Villar, C. d. (2012). En Liderazgo Empresarial. TLalnepplantla: Red Tercer Milenio.

Villacís B., (2010) En Censo 2010 Población y vivienda: una historia para ver y sentir. Instituto Nacional de Estadística y Censos (INEC). Quito - Ecuador. 